\title{
Direct Amidation of Esters via Ball Milling
}

\author{
William I. Nicholson, ${ }^{\mathrm{b}}$ Fabien Barreteau, ${ }^{\mathrm{c}}$ Jamie A. Leitch, ${ }^{\mathrm{a}}$ Riley Payne, ${ }^{\mathrm{a}}$ Ian Priestley, ${ }^{\mathrm{d}}$ Edouard \\ Godineau, ${ }^{,}$Claudio Battilocchio, ${ }^{* c}$ and Duncan L. Browne ${ }^{* a}$ \\ a Dr. Jamie A. Leitch, Riley Payne, Dr. Duncan L. Browne \\ Department of Pharmaceutical and Biological Chemistry, University College London (UCL), School of Pharmacy, 29-39 Brunswick Square, Bloomsbury, \\ London, WC1N 1AX, United Kingdom \\ E-mail: duncan.browne@ucl.ac.uk \\ b William I. Nicholson \\ School of Chemistry, Cardiff University, Main Building, Park Place, Cardiff, CF10 3AT, United Kingdom \\ c Dr. Fabien Barreteau, Dr. Edouard Godineau, Dr. Claudio Battilocchio \\ Syngenta Crop Protection AG, Rosentalstrasse 67, 4002 Basel, Switzerland \\ Email: claudio.battilocchio@syngenta.com \\ d Ian Priestley \\ Syngenta Ltd., Huddersfield Manufacturing Centre, Huddersfield, HD2 1FF, United Kingdom
}

\begin{abstract}
The direct mechanochemical amidation of esters - enabled by ball-milling - is herein described. The operationally simple procedure requires inputs of ester, amine, and sub-stoichiometric KOtBu and is applicable to a preparation of a large and diverse library of 78 amide structures with modest to excellent efficiency. Heteroaromatic and heterocyclic components are specifically shown to be amenable to this mechanochemical protocol. This direct synthesis platform has been applied to the synthesis of APl's, agrochemicals, and its ability to deliver gram-scale synthesis of active pharmaceuticals and building blocks is demonstrated, all in the absence of a reaction solvent.
\end{abstract}

\section{Introduction}

Despite the ubiquity of the amide linkage in biological, pharmaceutical, agrochemical, and natural product chemistry, the direct, efficient, and sustainable construction of amide bonds remains an often-overlooked synthetic challenge.$^{[1]}$ As highlighted in a study by Brown in 2014, as many as $50 \%$ of all medicinal chemistry routes contain at least one amide bond forming step. ${ }^{[2]}$ However the vast majority of these amides are constructed through the coupling of an amine with a carboxylic acid enabled by use of an activating reagent such as EDC, HATU, or oxalyl chloride (Scheme 1A). ${ }^{[3]}$ These established amidation protocols generate stoichiometric quantities of often toxic waste byproducts. ${ }^{[4]}$ This dichotomy has led the ACS green chemistry roundtable - initially in 2007, and again in 2018 - to highlight the requirement for more atom-economical approaches for forging this high-demand functional group i.e. without the need for stoichiometric reagents. A range of possible alternatives for the synthesis of amides have been developed utilizing organocatalysis, oxidative amidation, and the catalytic generation of activated carboxylates. ${ }^{[5]}$ To this end, one such avenue that has flourished is the direct amidation of carboxylic esters. ${ }^{[6]}$ Examples of powerful contemporary methods include - among many others - Newman and co-workers' $\mathrm{Ni}^{0}$-catalyzed direct and redox-neutral amidation of esters using $\mathrm{NHC}$ ligands ${ }^{[6 \mathrm{~m}, \mathrm{n}]}$ and Szostak and coworkers' direct amidation of simple esters using stoichiometric quantities of LHMDS (Scheme 1B). ${ }^{[60-q]}$

Recently our group have embarked on a synthesis programme centered around exploring concepts and opportunities offered by ball-milling. ${ }^{[7][8]}$ Through this strategy, there have been a wide array of synthetic organic transformations which have been explored by mechanochemistry, which, when analyzed as a whole reveal the synthetic opportunities enabled by the unique reactor environment of a milling-jar. ${ }^{[9]}$ Examples of these benefits include solvent-free reactions, reduced reaction times, alternative/improved reaction selectivity, and augmented/ complementary reaction efficiency. In the context of amidation by ball-milling whilst several protocols have already been reported, these methods centre around the translation of classical amide coupling reactions using diimide and uronium coupling reagents or activated starting materials in a mechanochemical environment. ${ }^{[10]}$ Accordingly, we recognized that a mechanochemical manifold - exploiting beneficial mass transfer afforded by ball-milling - that could enable the direct amidation of esters, negating the need for reaction solvent, transition-metal catalysts, or activating reagents could unveil significant and sustainable opportunities in synthetic route design, especially in industrial settings, and herein we wish to report our findings.

\section{Results and Discussion}

Our initial investigation into the mechanochemical direct amidation of esters employed ethyl benzoate (1a) and morpholine (2a) as model coupling partners (Scheme 1C). Using a mixer mill at $30 \mathrm{~Hz}$ for 1 hour, a survey of bases, of increasing strength, highlighted the notable activity of alkoxide bases in this reaction system (for full optimization details, see supporting information). Notably, no formation of the amide product was observed in the absence of base (entry 1). Potassium tert-butoxide was shown to be the most effective, leading to a promising $75 \%$ yield of the desired amide product (Scheme 1C, entry 3). Careful fine-tuning of the stoichiometry of base used in the reaction methodology (entries 4-6) revealed $85 \mathrm{~mol} \%$ of $\mathrm{KOtBu}$ to be the optimal balance ( $85 \%$ yield). ${ }^{[11]}$ Finally, increasing the equivalents of the ester from 1.0 to 1.2 provided complete conversion to the amide product in 1 hour with an almost quantitative isolated yield of $98 \%$ (entry 7). Notably, control experiments outside of the mechanochemical environment demonstrated substantially lower conversions in the same timeframe (entries 8-10). ${ }^{[12]}$

With optimal conditions in hand, the scope of ester starting materials was explored using morpholine as the amine component (Scheme 2A). Variation of the substitution pattern of the benzoate esters provided good to excellent yields for both electron-rich and -poor examples (55-98\% yield, 3a-j). Notably, and in stark difference to solvent based methods, the physical form of the starting material drastically alters the rate of reaction. 

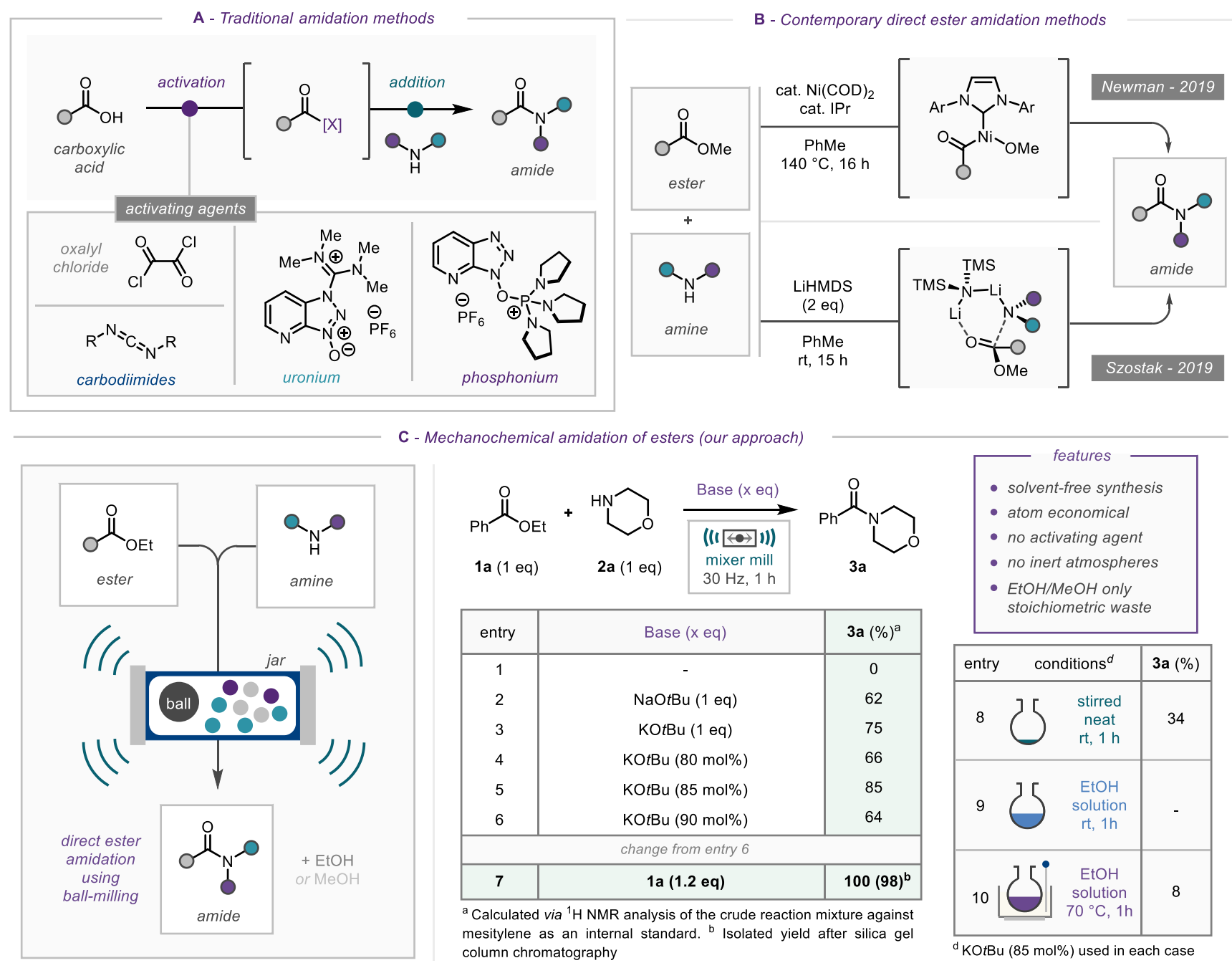

C - Mechanochemical amidation of esters (our approach)

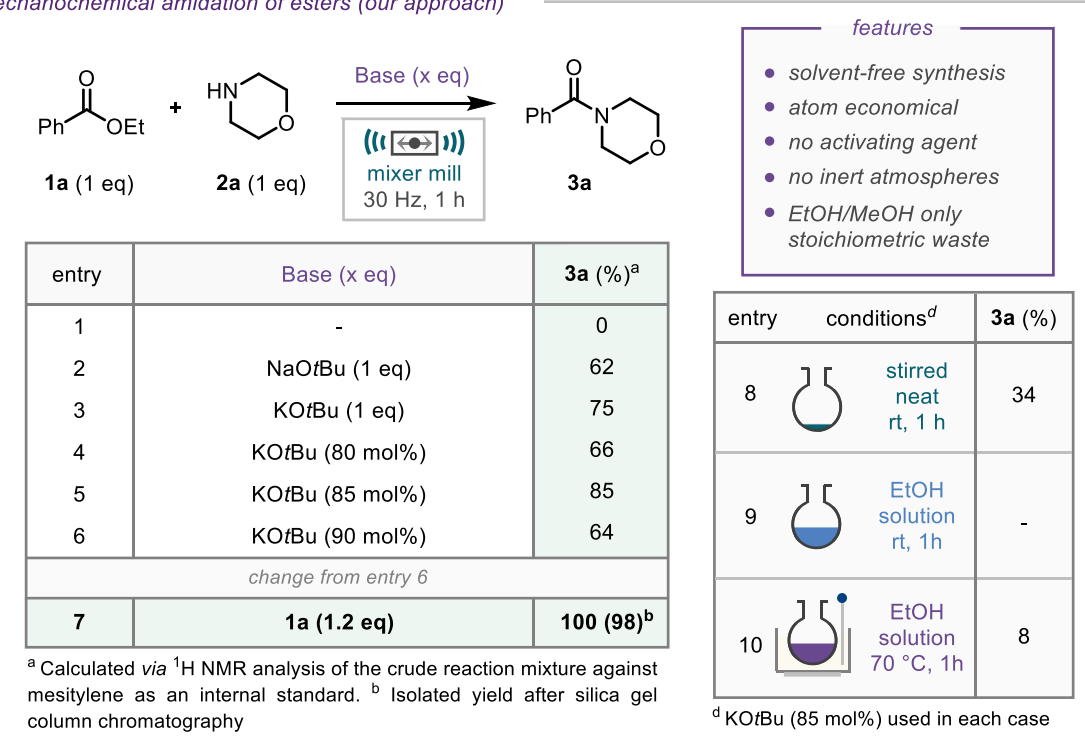

Scheme 1. Synthesis of amides via (A) amide coupling manifolds, (B) direct amidation of esters, (C) mechanochemical amidation of esters

For example, 4-bromo benzoate methyl ester, a solid at room temperature, and, 4-bromo benzoate ethyl ester, a liquid at room temperature, afforded the product in $42 \%$ and $80 \%$ respectively after 1 hour of reaction. Despite this, almost quantitative yield of the amide product was achieved when the reaction was carried out for 2 hours in the mill. In addition to simple aromatic esters, a range of alkyl esters including simple benzyl (3k), heterobenzyl (3l), and linear alkyl systems such as fatty acid derivatives proceeded with good efficiency $(\mathbf{3 m}-\mathbf{3 q})$. Furthermore, esters derived from amino acids - Boc- $(\mathrm{L})$-proline $(3 \mathbf{r}, 26 \%)^{[13]}$ and diethyl glycinate $(3 \mathrm{~s}, 82 \%)$ were compatible. A range of small carbocyclic and heterocyclic ring systems were smoothly introduced in the reaction system with modest to excellent efficiency enabling the introduction of cyclobutyl (3t, 68\%), azetidine $(3 \mathbf{y}, 80 \%)$, and protected isonipecotic acid derivatives (3w-x, 62-68\%). Interestingly, the successful ring opening of the lactone, benzofuran-2(3H)-one was also demonstrated in good yield $(\mathbf{3 z}, \mathbf{7 3} \%)$. Amidation of vinylic esters produced moderate to excellent yields (3aa-ab, 35-95\%).

The nature of the amine fragment was also studied using ethyl benzoate as the model ester and commenced by assessing cyclic secondary amines, similar to morpholine, including piperazine, piperidine, pyrrolidine, azepane and tetrahydroquiniline examples which all delivered the corresponding amide products in good to excellent yields (Scheme 2B, 3ac-ah, 49-98\%). Acyclic secondary amines also proved good coupling partners, although milling times were extended in this case to two hours to afford improved yields (3ai-ak). Primary aliphatic and benzylic amines also participate well in this direct transformation for example butylamine (3ar, 72\% yield) and 2-morpholinoethan-1-amine (3ap, $85 \%$ yield) provided the amide structure in good yields. More sterically demanding primary amine examples required increased reaction times (Scheme 2B, 3al 22\% and 3am 41\%). Additionally, a selection of aniline and amino-pyridine examples were also successfully coupled with ethyl benzoate (Scheme 2B, 3as-ba, $11-79 \%$ yield) including challenging bulky derivatives which often struggle under conditions using activated carboxylic acids.

Given the potential implications of this discovered reaction process to industrial discovery projects, we also looked to explore heteroaromatic ester and amine substrates. A range of ethyl or methyl esters containing pyridine, pyrazine, thiophene and benzofuran rings were all successfully coupled with morpholine (3bb-bh). Furthermore, in order to showcase the potential of this mechanochemical method in amide synthesis, a reactivity matrix was constructed using amine/aniline feedstocks and heteroaryl esters including pyridine and pyrazine (Scheme $2 \mathrm{C}$ ). 


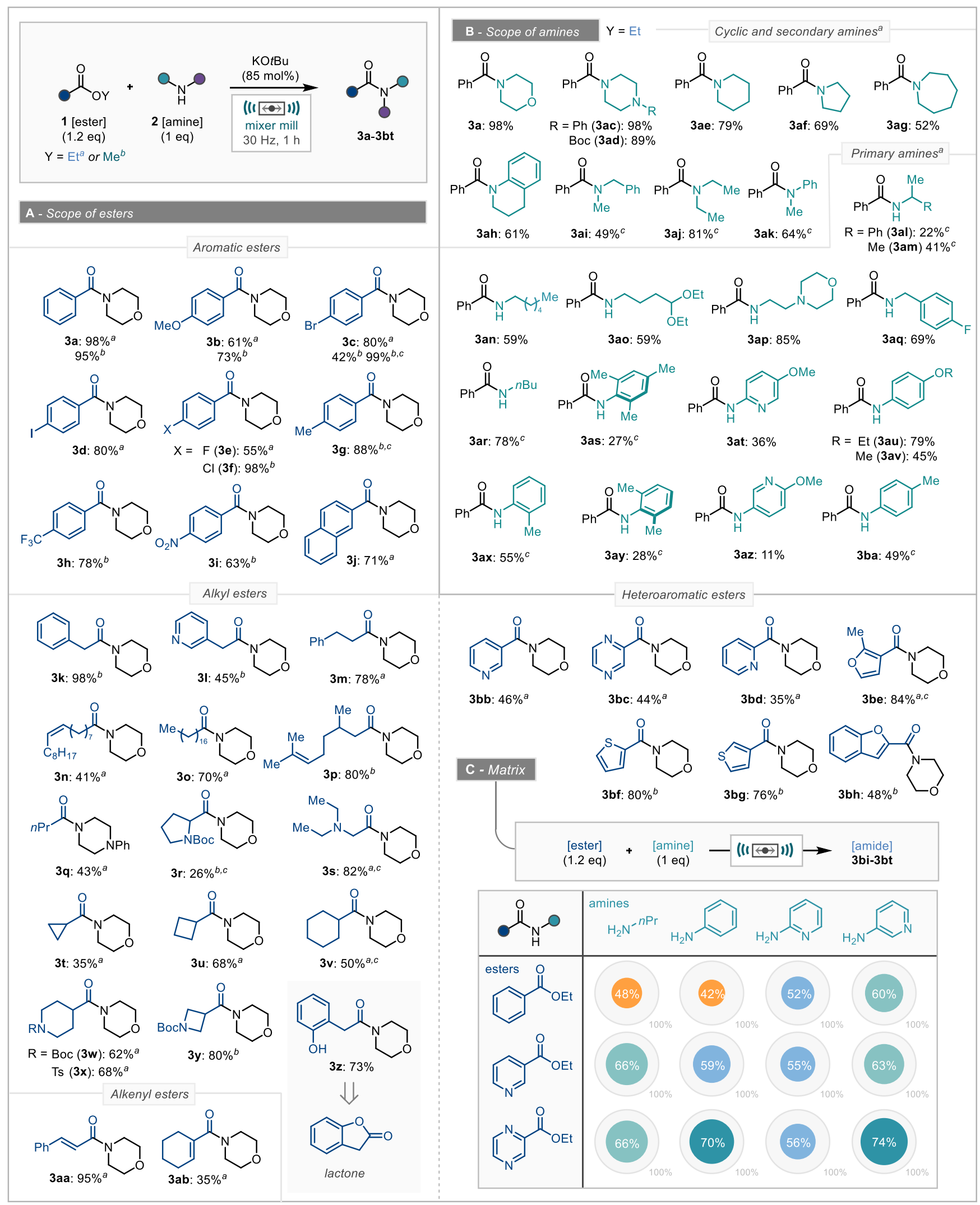

Scheme 2: Mechanochemical ester amidation via ball-milling. Reactions were carried out on $1 \mathrm{mmol}$ scale using a $14 / 15 \mathrm{~mL}$ stainless steel jar and a $4 \mathrm{~g}$ stainless steel ball (see supporting information for more details). ${ }^{a}$ Ethyl ester used. ${ }^{b}$ Methyl ester used. ${ }^{\mathrm{c}}$ Reaction time 2 hours 
In total, a collection of 12 compounds were prepared using this matrix manifold (3bi-3ct) in good to great yields, with higher efficiency observed with the pyrazine-containing structures.

Whilst ammonium salts are invariably solid, salts of this type are also often used as a stable equivalent for the long-term storage and/or transportation of amines. Conveniently it was found that ammonium salts were effective substrates for the direct amidation of esters by ball-milling, as long as an extra equivalent of potassium tert-butoxide base was added to the reaction mixture, with the resulting yields being on par or better than the free amine versions (Scheme 3A). Notably, application of ammonium chloride to these reaction conditions led to a promising unoptimized $16 \%$ isolated yield of primary amide $3 \mathrm{bu}$.

This reaction was also effective in the context of the direct synthesis of biologically relevant compounds including active pharmaceuticals and agrochemicals (Scheme 3B). In exploring these reactions, good to excellent yields were achieved for a broad range of compounds including, moclobemide (antidepressant, 3bv), lidocaine (anaesthetic, 3bz), coramine (stimulant, 3), fenfuram (fungicide, 3 by), and an analogue of CL82198, an MMP13 inhibitor (3ca).

This mechanochemical strategy was also directly scalable; use of a larger jar $(25 \mathrm{~mL})$ and heavier milling ball $(12 \mathrm{~g})$ and utilizing the capability of milling two reaction jars simultaneously the reaction could be successfully scaled (10-fold) to produce $2.4 \mathrm{~g}$ ( $90 \%$ yield) of moclobemide in one hour. Further expanding this, using two $30 \mathrm{~mL}$ jars with a $16 \mathrm{~g}$ ball in each, the model reaction between ethyl benzoate and morpholine delivered $3.21 \mathrm{~g} \mathrm{(83 \% )}$ of the corresponding amide (3a) in 2 hours of milling, representing a 20 -fold scale up.

With regards to assessing the green metrics of our reaction process compared to more traditional approaches, a simple atom economy calculation ( $\mathrm{M}_{\mathrm{r}}$ of inputs/ $\mathrm{M}_{\mathrm{r}}$ of the product) demonstrates the process to be quite favourable at $55 \%$, compared to a phosgene activation of carboxylic acids at $47 \%$ (Scheme 3D, see supporting information for further details). ${ }^{[14]}$

\section{Conclusion}

In conclusion, the direct amidation of esters can be readily achieved by ball-milling without the requirement for bulk reaction solvent. This reaction process is applicable to a very broad scope of aryl and alkyl ester substrates, along with an array of secondary and primary amines. Furthermore, this concept was showcased in the matrix synthesis of a library of heteroaromatic amide derivatives, the application to ammonium salts and in the synthesis of a number of biologically relevant compounds including active pharmaceuticals and agrochemicals.
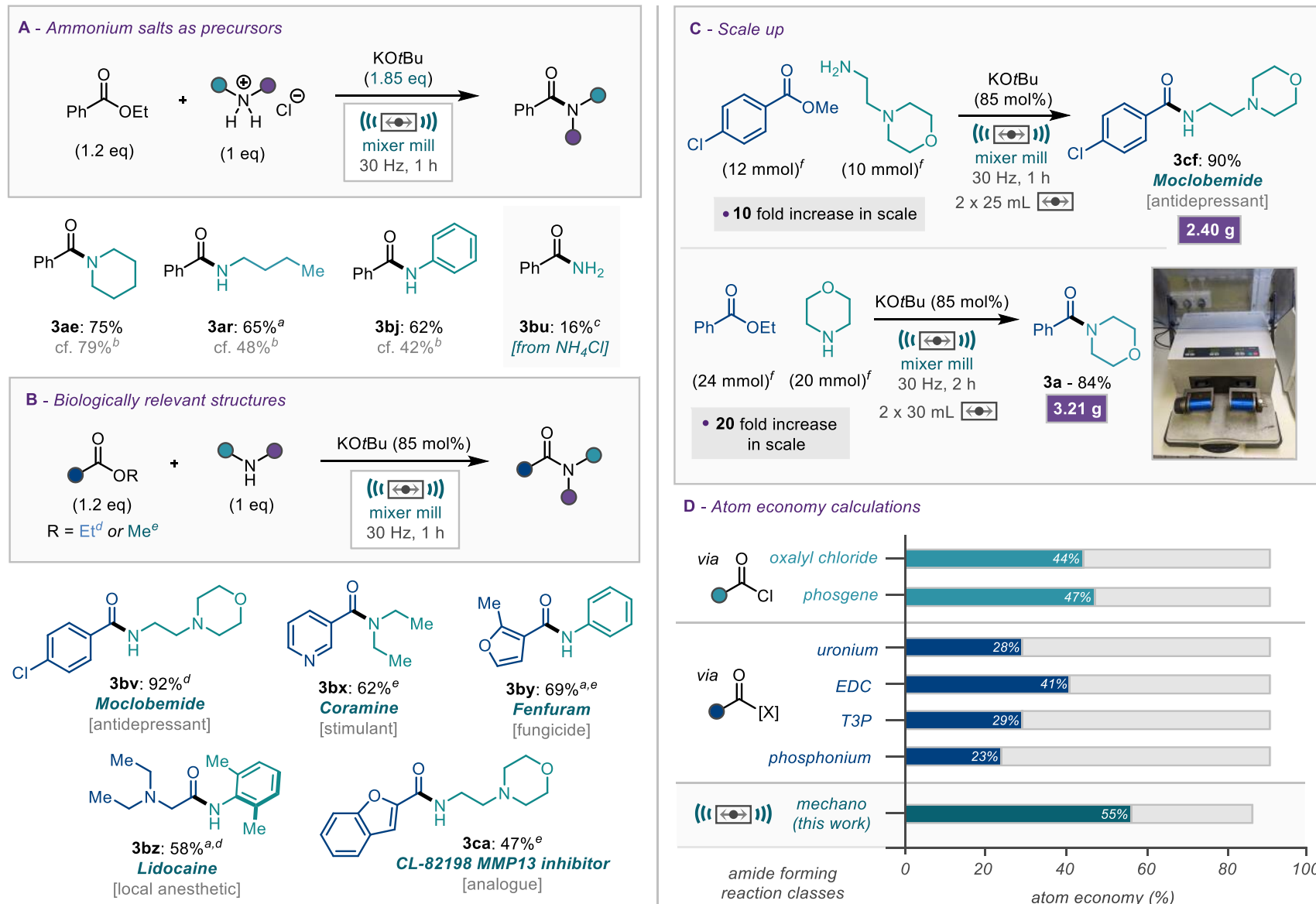

D - Atom economy calculations

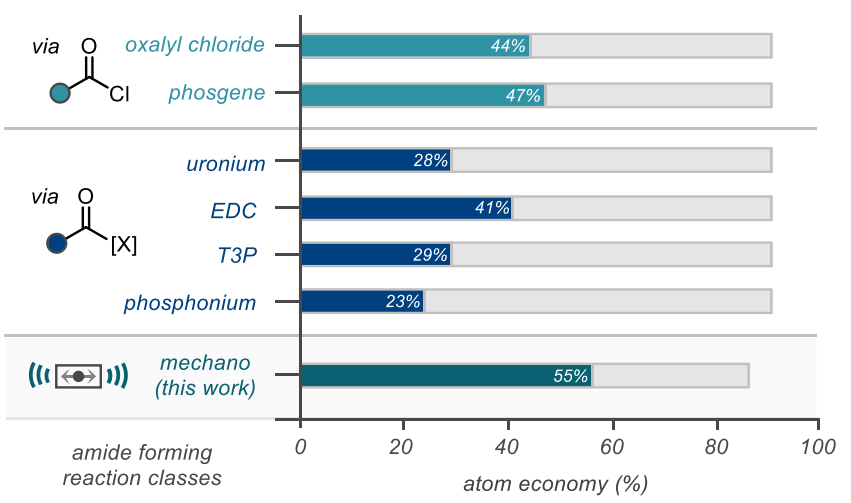

Scheme 3: Mechanochemical ester amidation. (A) use of ammonium salts in the reaction methodology. (B) Synthesis of biologically-relevant amides, (C) scale-up procedures, (D) atom economy calculations. ${ }^{a}$ Reaction time 2 hours. ${ }^{b}$ From Scheme 2. ${ }^{c} 2 a(1 \mathrm{eq}), \mathrm{NH}_{4} \mathrm{Cl}(2 \mathrm{eq}), \mathrm{KOtBu}(2.85 \mathrm{eq}) .{ }^{\mathrm{d}}$ Ethyl ester was used. ${ }^{\mathrm{e}} \mathrm{Methyl}$ ester was used. ${ }^{f}$ Two jars with half material in each. 
Moreover, the mechanochemical amidation protocol was scaled up to produce almost $2.5 \mathrm{~g}$ of the antidepressant moclobemide, and up to $3.2 \mathrm{~g}$ of the model amide structure $3 \mathrm{a}$. This sustainable, mild, atom-economical, and efficient amidation protocol will not only spark interest but have direct implications in the application of ball milling and other mechanochemical synthesis techniques in an industrial setting.

\section{Acknowledgements}

W.I.N. thanks Cardiff University for a studentship. J.A.L. thanks the Leverhulme Trust for a research fellowship (RPG-2019-260). We thank Prof. Tom Sheppard (UCL) for helpful discussions. The authors would like to thank Gavin Bluck, Andrei losub, George Hodges, Faima Lazreg, Jean-Philippe Krieger, Kenneth Ling, Christophe Grojean, Alan Robinson, and Maria Ciaccia for valuable discussion.

Keywords: mechanochemistry $\cdot$ amides $\cdot$ amidation $\bullet$ ballmilling

[1] a) V. R. Pattabiraman, J. W. Bode, Nature, 2011, 480, 471 - 479; b) M. Benaglia, M. Pirola, E. Massolo, Eur. J. Org. Chem. 2020, 2020, 4641 4651 .

[2] J. Boström, D. G. Brown, J. Med. Chem. 2016, 59, 4443 - 4458

[3] a) M. T. Sabatini, L. T. Boulton, H. F. Sneddon, T. D. Sheppard, Nature Catalysis 2019, 2, $10-17$; c) N. Schneider, D. M. Lowe, R. A. Sayle, M. A. Tarselli, G. A. Landrum, J. Med. Chem. 2016, 59, 4385-4402; d) J. R. Dunetz, J. Magano, G. A. Weisenburger, Org. Process Res. Dev. 2016 $20,140-177$

[4] a) M. P. Thompson, I. Peñafiel, S. C. Cosgrove, N. J. Turner, Org. Process Res. Dev. 2019, 23, 9 - 18; b) J. B. Sperry, C. J. Minteer, J. Y. Tao, R. Johnson, R. Duzguner, M. Hawksworth, S. Oke, P. F. Richardson R. Barnhart, D. R. Bill, R. A. Giusto, J. D. Weaver III, Org. Process Res. Dev. 2018, 22, 1262 - 1275; c) D. J. C. Constable, P. J. Dunn, J. D. Hayler, G. R. Humphrey, J. L. Leazer, Jr, R. J. Lindermann, K. Lorenz, J. Manley, B. A. Pearlman, A. Wells, A. Zaks, T. Y. Zhang, Green Chem. 2007, 9, 411 - 420; d) M. C. Bryan, P. J. Dunn, D. Entwistle, F. Gallou, S. G. Koenig, J. D. Hayler M. R. Hickey, S. Hughes, M. E. Kopach, G. Moine, P. Richardson, F. Roschangar, A. Steven, F. J. Weiberth, Green Chem. 2018, 20, $5082-5103$.

[5] For reviews on amide-forming reactions, see: a) E. Valeur, M. Bradley, Chem. Soc. Rev. 2008, 38, 606-631; b) R. M. Lanigan, T. D. Sheppard, Eur. J. Org. Chem. 2013, 2013, $7453-7465$. c) C. L. Allen, J. M. J. Williams, Chem. Soc. Rev. 2011, 40, 3405-3415; d) R. M. de Figueiredo J.-S. Suppo, J.-M. Campagne, Chem. Rev. 2016, 116, $12029-12122$ e) H. Lundberg, F. Tinnis, N. Selander, H. Adolfsson, Chem. Soc. Rev. 2014, 43, 2714 - 2742; e) M. Todorovic, D. M. Perrin, Peptide Science 2020, 112, e24210; For selected examples of amidation protocols, see: f) K. Ishihara, S. Ohara, H. Yamamoto, J. Org. Chem. 1996, 61, $4196-$ 4197; g) T, Marcelli, Angew. Chem. 2010, 122, 6992 - 6995; Angew. Chem. Int. Ed. 2010, 49, 6840 - 6843; h) B. Shen, D. M. Makley, J. N. Johnston, Nature 2010, 465, 1027 - 1932; i) P. Starkov, T. D. Sheppard Org. Biomol. Chem. 2010, 9, 1320 - 1323; j) R. M. Lanigan, P. Starkov, T. D. Sheppard, J. Org. Chem. 2013, 78, 4512 - 4523; k) P. Tang, Org. Synth. 2009, 81, 262 - 272; I) M. T. Sabatini, L. T. Boulton, T. D. Shepphard, Sci. Adv. 2017, 3, e1701028; m) J. W. Bode, S. S. Sohn, J Am. Chem. Soc. 2007, 129, $13798-13799 ; n)$ H. U. Vora, T. Rovis, J. Am. Chem. Soc. 2007, 129, $13796-13797$; o) W.-J. Yoo, C.-J. Li, J. Am Chem. Soc. 2006, 128, $13064-13065$; p) C. Gunanathan, Y. Ben-David, D. Milstein, Science 2007, 317, 790 - 792; q) L. U. Nordstrøm, H. Vogt R. Madsen, J. Am. Chem. Soc. 2008, 130,17672-17673; r) W.-K. Chan C.-M. Ho, M.-K. Wong, C.-M. Che, J. Am. Chem. Soc. 2006, 128, 14796 - 14797; s) W.-J. Yoo, C.-J. Li, J. Am. Chem. Soc. 2006, 128, $13064-$ 13065; t) X. Li, S. J. Danishefsky, J. Am. Chem. Soc. 2008, 130, $5446-$ 5448; u) T. Krause, S. Baader, B. Erb, L. J. Gooßen, Nat. Commun. 2016 7, 11732; v) H. Lundberg, F. Tinnis, J. Zhang, A. G. Algarra, F. Himo, H. Adolfsson, J. Am. Chem. Soc. 2017, 139, 2286-2295; x) Z. Liu, J. Zhang, S. Chen, E. Shi, Y. Xu, X. Wan, Angew. Chem. 2012, 124, $3285-3289$; Angew. Chem. Int. Ed. 2012, 51, 3231 - 3235; y) N. Caldwell, C. Jamieson, I. Simpson, A. J. B. Watson, Chem. Commun. 2015, 51, 9495 -9498; z) C. G. McPherson, N. Caldwell, C. Jamieson, I. Simpson, A. J. B. Watson, Org. Biomol. Chem. 2017, 15, 3507 - 3518; Also see reviews in reference [1].

[6] For some examples of the direct amidation of esters see: a) A. Basha, $M$ Lipton, S. M. Weinreb, Tetrahedron Lett. 1977, 18, 4171 - 4172; b) T. Gustafsson, F. Pontén, P. H. Seeberger, Chem. Commun. 2008, 44 $1100-1102$; c) C. Ferroud, M. Godart, S. Ung, H. Borderies, A. Guy, Tetrahedron Lett. 2008, 49, 3004 - 3008; d) B. R. Kim, H.-G. Lee, S.-B. Kang, G. H. Sung, J.-J. Kim, J. K. Park, S.-G. Lee, Y.-J. Yoon, Synthesis 2012, 1, 42 - 50; e) J. L. Vrijdag, F. Delgado, N. Alonso, W. M. De
Borggraeve, N. Pérez-Macias, J. Alcázar, Chem. Commun. 2014, 50 15094 - 15097; f) T. Ooi, E. Tayama, M. Yamada, K. Maruoka, Synlett, 1999, 6, 729-730; f) T. Ohshima, Y. Hayashi, K. Agura, Y. Fujii, A Yoshiyama, K. Mashima, Chem. Commun. 2012, 48, 5434-5436; g) H. Morimoto, R. Fujiwara, Y. Shimizu, K. Morisaki, T. Ohshima, Org. Lett 2014, 16, 2018-2021; h) A. Ali, S. M. A. H. Siddiki, K. Kon, K.-I. Shimizu, Chem CatChem 2015, 7, 2705 - 2710; i) C. W. Cheung, M. L. Ploeger, X. Hu, Nat. Commun. 2017, 8, 14878; j) B. D. Mkhonazi, M. Shandu, R Tshinavhe, S. B. Simelane, P. T. Moshapo, Molecules, 2020, 25, 1040 k) N. Rashed, K. Masuda, T. Ichitsuka, N. Koumura, K. Sato, S. Kobayashi, Adv. Synth. Catal. 2021, DOI:10.1002/adsc.202001496; I) Z. Fu, X. Wang, S. Tao, Q. Bu, D. Wei, N. Liu, J. Org. Chem. 2021, 86, 2339 $-2358 ; \mathrm{m})$ T. B. Halima, J. Masson-Makdissi, S. G. Newman, Angew. Chem. 2018, 130, 13107 - 13111; Angew. Chem. Int. Ed. 2018, 57 12925 - 12929; n) Y.-L. Zheng, S. G. Newman ACS Catal. 2019, 9, 5 4426 - 4433; o) G. Li, C.-L. Ji, X. Hong, M. Szostak, J. Am. Chem. Soc. 2019, 141, 11161 - 11172; p) G. Li, M. Szostak, Nat. Commun. 2018, 9, 4165; q) M. Rahman, G. Li, M. Szostak, J. Org. Chem. 2019, 84, 12091 -12100 .

[7] For some recent examples of synthetic reactions by mechanochemistry, see: a) K. Kubota, Y. Pang, A. Miura, H. Ito, Science, 2019, 366, $1500-$ 1504; b) C. Schumacher, J. G. Hernández, C. Bolm, Angew. Chem. 2020, 132, 16499 - 16502; Angew. Chem. Int. Ed. 2020, 59, 16357 - 16360; c) Y. Pang, J. W. Lee, K. Kubota, H. Ito, Angew. Chem. 2020, 132, 22759 - 22765: Angew. Chem Int Ed 2020, 59, 22570 - 22576; d) T. Seo, N. Toyoshima, K. Kubota, H. Ito, J. Am. Chem. Soc. 2021, 143, $6165-$ 6175; e) K. J. Ardila-Fierro, S. Lukin, M. Etter, K. Užarević, I. Halasz, C Bolm, J. G. Hernández, Angew. Chem. 2020, 132, 13560 - 13564 Angew. Chem. Int. Ed. 2020, 59, 13458 - 13462; f) J. Anderson, J. Mack, Angew. Chem. 2018, 130, 13246 - 13249; Angew. Chem. Int. Ed. 2018, 57, $13062-13065 ;$ g) S. Ni, M. Hribersek, S. K. Baddigam, F. J. L. Ingner, A. Orthaber, P. J. Gates, L. T. Pilarski, Angew. Chem. 2020, 133, 6734 - 6740; Angew. Chem. Int. Ed. 2020, 60,6660 - 6666; h) Q. Cao, D. E. Crawford, C. Shi, S. L James, Angew. Chem. 2020, 132, 4508 - 4513; Angew. Chem. Int. Ed. 2020, 59, 4478 - 4483; i) C. Bolm, R. Mocci, C Schumacher, M. Turberg, F. Puccetti, J. G. Hernández, Angew. Chem. 2018, 130, 2447 - 2450; Angew. Chem. Int. Ed. 2018, 57, 2423 - 2426.

a) M. T. J. Williams, L. C. Morrill, D. L. Browne, ACS Sustainable Chem. Eng. 2020, 8, $17876-17881$; b) W. I. Nicholson, A. C. Seastram, S. A lqbal, B. G. Reed-Berendt, L. C. Morrill, D. L. Browne, ChemSusChem. 2020, 13, 131 - 135; c) A. C. Jones, W. I. Nicholson, H. R. Smallman, D. L. Browne, Org. Lett. 2020, 22, 7433 - 7438; d) Q. Cao, W. I. Nicholson, A. C. Jones, D. L. Browne, Org. Biomol. Chem 2019, 17, 1722 - 1726; e) Q. Cao, J. L. Howard, E. Wheatley, D. L. Browne, Angew. Chem. 2018, 130, 11509 - 11513; Angew. Chem. Int. Ed. 2018, 57, 11339 - 11343; f J. L. Howard, W. I. Nicholson, Y. Sagatov, D. L. Browne, Beilstein J. Org. Chem. 2017, 13, 1950 - 1956; g) J. L. Howard, M. C. Brand, D. L. Browne, Angew. Chem. 2018, 130, 16336 - 16340; Angew. Chem. Int. Ed. 2018, 57, $16104-16108$.

[9] a) J. L. Howard, Q. Cao, D. L. Browne, Chem. Sci. 2018, 9, 3080 - 3094 b) J.-L. Do, T. Friščić, ACS Cent. Sci. 2017, 3, 13 - 19; c) J. G. Hernandez, C. Bolm, J. Org. Chem. 2017, 82, 4007 - 4019; d) O. Eguaogie, J. S. Vyle, P. F. Conlon, M. A. Gîlea, Y. Liang, Beilstein J. Org Chem. 2018, 14, 955 - 970; e) T.-X. Metro, J.' Martinez, F. Lamaty, ACS Sustainable Chem. Eng. 2017, 5, 9599 - 9602; f) S. L. James, C. J. Adams, C. Bolm, D. Braga, P. Collier, T. Frisčič, F. Grepioni, K. D. M. Harris, G. Hyett, W. Jones, A. Krebs, J. Mack, L. Maini, A. G. Orpen, I. P. Parkin, W. C. Shearouse, J. W. Steed, D. C. Waddell, Chem. Soc. Rev. 2012, 41, 413 - 447; g) F. Gomollón-Bel, Chemistry International, 2019 $41,12-17$.

[10] a) V. Declerck, P. Nun, J. Martinez, F. Lamaty, Angew. Chem. 2009, 121, 9482 - 9485; Angew. Chem. Int. Ed. 2009, 48, 9318-9321; b) G. Kaupp, J. Schmeyers. J. Boy, Tetrahedron, 2000, 56, 6899 - 6911; c) J. G. Hernández, E. Juaristi, J. Org. Chem. 2010, 75, 7107 - 7111; d) T.-X Métro, J. Bonnamour, T. Reidon, J. Sarpoulet, J. Martinez, F. Lamaty, Chem. Commun. 2012, 48, 11781 - 11783; e) V. Štrukil, B. Bartolec, T. Portada, I. Đilović, I. Halasz, D. Margetić, Chem. Commun. 2012, 48, 12100 - 12102; f) J. Bonnamour, T.-X. Métro, J. Martinez, F. Lamaty, Green Chem. 2013, 15, 1116 - 1120; g) C. Duangkamol, S. Jaita, S. Wangngae, W. Phakhodee, M. Pattarawarapan, RSC Adv. 2015, 5 52624 - 52628; h) V. Porte, M. Thioloy, T. Pigoux, T.-X. Métro, J. Martinez, F. Lamaty, Eur. J. Org. Chem, 2016, 2016, 3505 - 3508; i) D. Margetić, V. Štrukil, in Mechanochemical Organic Synthesis, Chapter 3 - Carbon-Nitrogen Bond-Formation Reactions, Elsevier, 2016, pp. 141 233; j) J. G. Hernández, K. J. Ardila-Fierro, D. Crawford, S. L. James, C Bolm, Green Chem, 2017, 19, 2620 - 2625; k) L. Gonnet, T. Tintillier, N. Venturini, L. Konnert, J.-F. Hernandez, F. Lamaty, G. Laconde, J. Martinez, E. Colacino, ACS Sustainable Chem. Eng, 2017, 5, $2936-$ 2941 ; I) K. J. Ardila-Fierro, D. E. Crawford, A. Körner, S. L. James, C Bolm, G. J. Hernández, Green Chem. 2018, 20, 1262 - 1269; m) Y Yeboue, B. Gallard, N. Le Moigne, M. Jean, F. Lamaty, J. Martinez, T. X. Métro, ACS Sustainable Chem. Eng, 2018, 6, 16001 - 16004; n) N. Cindro, M. Tireli, B. Karadeniz, T. Mrla, K. Užarević, ACS Sustainable Chem. Eng, 2019, 7, 16301 - 16309; o) E. Broumidis, M. C. Jones, F. Vilela, G. O. Lloyd, ChemPlusChem, 2020, 85, 1754 - 1761; p) T. Dalidovich, K. A. Mishra, T. Shalima, M. Kudrjašova, D. G. Kananovich, R. Aav, ACS Sustainable Chem. Eng, 2020, 8, 15703 - 15715; q) Y. Yeboue, M. Jean, G. Subra, J. Martinez, F. Lamaty, T.-X. Métro, Org Lett. 2021, 23, 631 - 635; r) R. Mocci, E. Colacino, L. De Luca, C. Fattuoni, A. Porcheddu, F. Delogu, ACS Sustainable Chem. Eng. 2021 
9, $2100-2114$; s) T. Stolar, S. Grubešić, N. Cindro, E. Meštrović, K. Užarević, J. G. Hernández, Angew. Chem. 2021 10.1002/ange.202100806; Angew. Int, Ed. 2021, 10.1002/anie.202100806.

[11] It remains unclear why 0.85 equivalents of base are optimal, howeve this optimum value has been confirmed in three different laboratories.

[12] During finalization of this manuscript, Cheng, Chen, and Qin reported a solvent-free direct amidation under similar conditions to those in entry 8 see: (a) R. Zhang, W.-Z. Yao, L. Qian, W. Sang, Y. Yuan, M.-C. Du, H
Cheng, C. Chen, X. Qin, Green Chem. 2021, DOI: 10.1039/D1GC00720C

[13] Complete erosion of the enantioenriched $\alpha$-stereocentre derived from enantiopure Boc-Pro-OMe was observed under the reaction conditions.

[14] a) B. M. Trost, Science 1991, 254, 1471 - 1477; b) B. M. Trost, Angew. Chem. Int. Ed. 1995, 34, 259 - 281; c) P. Anastas, N. Eghbali, Chem. Soc. Rev. 2010, 39, $301-312$; d) B. M. Trost, Acc. Chem. Res. 2002 , 35, 695-705; e) R. A. Sheldon, Chem. Soc. Rev. 2012, 41, 1437-1451; e) T. Newhouse, P. S. Baran, R. W. Hoffmann, Chem. Soc. Rev. 2009 $38,3010-3021$. 